\title{
Electromagnetic Field Detector Circuit for Low- Frequency Energy Applications
}

\author{
M.M. Bait-Suwailam \\ Department of Electrical and Computer Engineering, Sultan Qaboos University, P.O. Box 33, PC 123, Muscat, Oman
}

Received 7 September 2014; accepted 11 January 2015

\begin{abstract}
This study details an electromagnetic (EM) field radiation detector system that was developed for near-field low-frequency energy applications. The prototype constitutes the use of a dual-band monopole antenna system as a probe along with a detecting circuit. Furthermore, the prototype was equipped with a qualitative EM radiation strength display unit at its output stage. For proof of concept, the detecting probe was implemented on a printed-circuit board. Both numerical simulations were based on PSpice software (Cadence Design Systems, Inc., San Jose, California, USA) and measurements are presented and discussed. The EM field detector aimed to sense any potential sources of EM radiation from mobile phone units as well as WiFi access points, simultaneously, which is accomplished with the use of the dual-band antenna system. Such a sensitive detector has useful application as a stand-alone monitoring probe for troubleshooting as well as to identify sources of EM radiation interference threats for industrial high-speed electronic devices. Additionally, such a sensor is a potentially useful tool for site testing and scanning for optimal locations of base station masks for telecommunication service providers. Other prototypes are also presented to illustrate the usefulness of such detectors in some of the aforementioned applications.
\end{abstract}

Keywords: Antenna, Electromagnetic radiation, Detector circuit, Probe, Sensor.

\section{دائرة للكشف عن المجال الكهرومغناطيسي لتطبيقات الطاقة المنخفضة التردد}

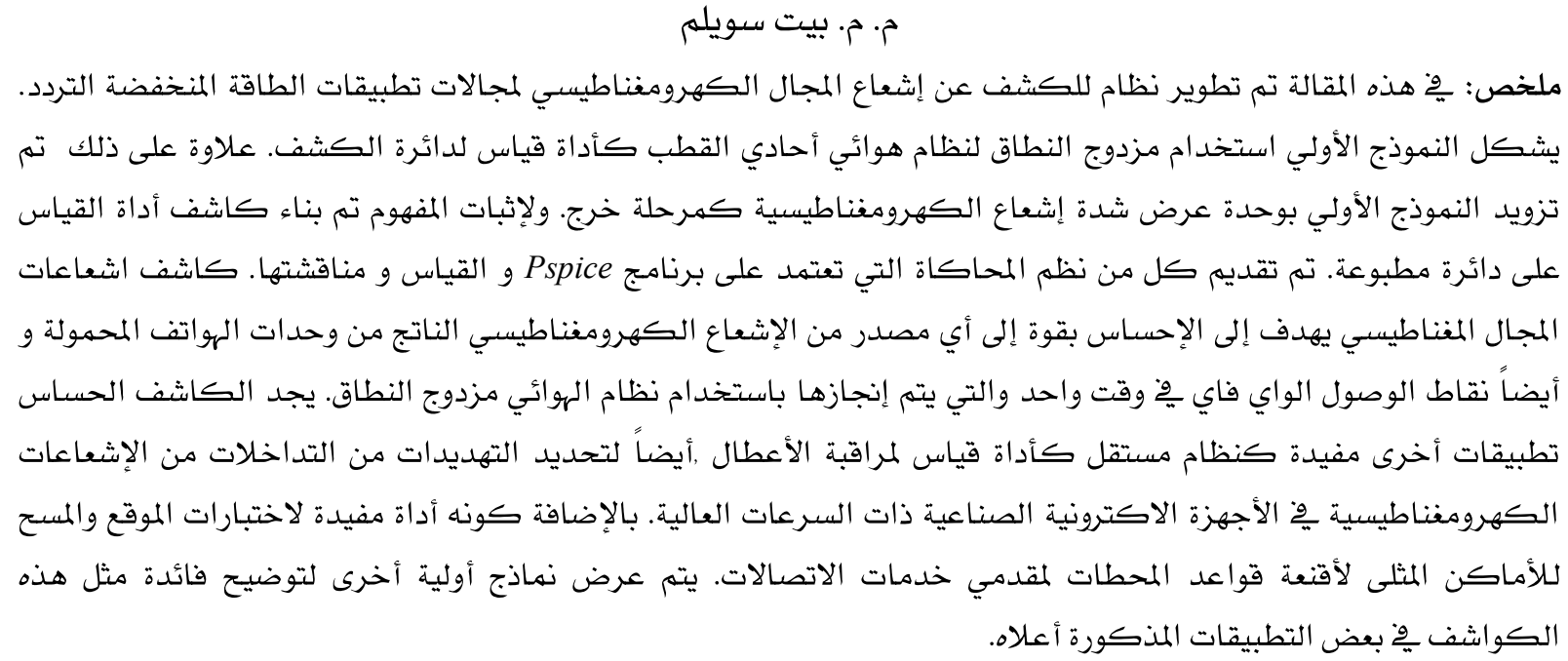

Corresponding author's e-mail: msuwailem@squ.edu.om 


\section{Introduction}

In the atmospheric space around us, there are various types of electromagnetic radiation which are present due to the operation of high-speed electronic devices, microwave systems / subsystems, and telecommunication devices, as well as the use of radio frequency (RF). With the rapid growth in the wireless, industrial, and medical sectors nowadays, there has been an increase in the presence and use of mobile communication devices. Additionally, the excessive installation of high-powered base station towers, ubiquitous electronic devices and sensors, and the constant use of broadcast radio and TV transmitters, and satellite systems have led to public concern. This concern stems from the possibility of hazardous and adverse health effects associated with exposure to RF radiation originating from such systems and high-speed devices (Advisory Group on Non-ionizing Radiation, 1992; Advisory Group on Non-ionizing Radiation, 1994; Hanna et al. 2009; International Commission on Non-Ionizing Radiation Protection, 1998; IEEE Standards Association, 1994; Mann et al. 2001; World Health Organization, 2011). Hence, there is a need for monitoring systems that can detect and predict sources of EM radiation along with their energy strength. It is well known from classical physics theory that electromagnetic (EM) waves can propagate and travel at the speed of light in free space or slower than the speed of light in any other respective media. Such waves behave as plane waves when far away from the source of radiation, in which the electric field, magnetic field, and direction of propagation form a mutual orthogonal triplet. In fact, as frequency of the propagating wave is increased, EM waves have strong energy levels which may be hazardous.

In general, the human eye can detect light in free space at wavelengths between 400-700 nanometers (Ulaby et al. 2014). Hence, this spectrum range is known as visible light. However, the objects around us can also emit other types of EM radiation that cannot be seen by the human eye, such as RF waves. Such EM radiation exists in the universe surrounding $u$ s.

Furthermore, the energy carried by EM waves emanating from mobile devices can easily interfere with other devices that operate within close proximity to the operating frequency of such mobile devices, for instance, around the GSM-900 megahertz (MHz) frequency band.

Another example of an originator of EMradiated emissions is conductive coupling paths. For example, connectors, like wires and cables, radiate at a high frequency; thus, their EM wavelengths cannot be neglected for their contribution in increasing frequency so that it is close to the GHz range. Such is the case of today's connectors and accessories for high-speed electronic devices, such as power cables (Archambeault et al. 2001; Bait-Suwailam et al. 2014; Brench and Brench 1989; Buchanan et al. 1994; Park et al. 2013). In sum, EM waves are everywhere and cannot be ignored. Their strength and existence will dramatically increase in the coming years with the rapid growth of technology and employed services. An example of this is, 4G service, which is available at present, and the upcoming 5G service, which will no doubt result in an increase in the number of base station towers and an additional increase in their power strength in order to provide broadband mobile services to customers (Datang Telecom Technology \& Industry Group, 2013).

In order to provide satisfactory safety margins for the public, several international organizations worldwide have set the health and safety guidelines which are currently in place. These guidelines' ability to limit public exposure to radio waves from base stations and mobile phones are well documented, having been set by the International Commission on Non-Ionizing Radiation Protection (ICNIRP) (1998).

The amount of EM radio wave energy absorbed by the human body is quantified as specific absorption rate (SAR). In practice, mobile devices and high-speed electronic devices, for instance touch pads, are assessed for potentially excessive of EM radiation by a series of SAR measurements prior to certification in order to meet the Federal Communications Commission (FCC) regulations (ICNIRP, 1998). The spatial peak SAR limit set by the FCC in the US is $1.6 \mathrm{~W} / \mathrm{kg}$ over 1 gram of actual tissue, while it is $2 \mathrm{~W} / \mathrm{kg}$ in Europe (Bernardi et al. 1998; Rowley and Waterhouse 1999; Seabury 2005). Although knowledge of SAR levels and appliances' EM output is important, the public generally lacks knowledge of these limits, and compatibility testing equipment is not easily found. Therefore, it is believed that alternative, easy-to-find-and-use techniques would attract 
public attention. Such alternatives rely on developing highly sensitive EM-detecting units that could be of interest in identifying potential sources of electromagnetic radiation interference EMI). Hence, an electrician, or even a homeowner, might employ this device to locate buried electrical lines or even defects within high-speed electronic devices.

In (Lin, James 2009), EM radiation detectors using electronic circuitry were briefly developed. Herein, further results covering applications to detect malfunctions in power meters as well as high-speed electronic circuits are presented. Based on qualitative results, the probes are found to be highly sensitive to any EM radiation within lowfrequency range. It is believed that the stand-alone detector circuit will also be useful as a measurement tool to predict EM radiated emissions from high-speed chips/circuits, especially for certification of electronic devices in industries as well as in medical sector. In addition to the aforementioned applications, such detector is of no doubt beneficial to predict any unauthorized use of mobile phones in examination halls in academia as well as in security rooms. This paper is organized as follows. Section 2 covers details of the developed detector circuit that targets three applications, namely: power line meters, extremely low-frequency energy targeting printed circuit boards, and lowfrequency regime covering mobile phone devices and WiFi access points. Both simulation and experimental results are then presented and discussed in section 3 . Section 4 concludes with a brief summary of the findings.

\section{The Developed Detector}

The detecting prototype that is developed here constitutes the use of an antenna system, followed with a detecting circuitry and ends up with an indicator (LED) as well as a qualitative field strength display unit. Figure 1 shows block diagram of the aforementioned stages of the developed detector.

At an early stage through the design and numerical simulations, a pre-defined alternating current (AC) signal was used instead of the antenna system to mimic an incoming electromagnetic wave. Note that in all the simulated detectors, an AC voltage source used as an input to mimic the detected electromagnetic wave captured by the antenna. In this research work, a quarter-wavelength $(\lambda / 4)$ thin metallic monopole antenna is considered in all prototypes. A view of such antenna is shown in Fig. 2, where the chassis is used as a ground plane for the antenna. The resonance frequency of the monopole antenna is determined by its length $d$, which is given by:

$$
d=\lambda_{0} / 4
$$

where $\lambda_{0}$ is the free-space wavelength of the electromagnetic wave at the operating frequency. Note that in practice, the resonance frequency will in fact need to be slightly less than a quarterwavelength.

An added advantage of the use of monopole antennas is their omnidirectional radiation pattern in the horizontal plane (H-plane), in which the antenna will be able to detect incoming electromagnetic waves arriving from any direction (Balanis, C. 2005). The radiation pattern of monopole antennas has a typical donut shape in the vertical plane (E-plane). In order to identify the resonance of the monopole antenna, the reflection coefficient (or standing wave ratio SWR) is studied. Figure 3 shows the voltage SWR for one of the vertical monopole antenna that resonates within the $900 \mathrm{MHz}$ band, where the electrical length of the antenna is nearly $8.3 \mathrm{~cm}$.

It is essential to highlight here that several design constraints and challenges were encountered, including mostly the employed active devices, for instance operational amplifier and transistors. Such components were found to be band limited and sensitive to certain operating frequency range. As such, three prototypes were developed targeting several applications, with emphasis on the radiation out from mobile devices and WiFi access points. The detecting prototypes are discussed and presented next.

\section{Numerical Results and Experimental Validation}

\subsection{Detectors for Power Transmission Line Meters}

Figure 4 depicts the developed detector circuit. For simulation purposes, a pre-defined AC signal was assumed in order to mimic an incoming radiated $\mathrm{EM}$ wave in space. 


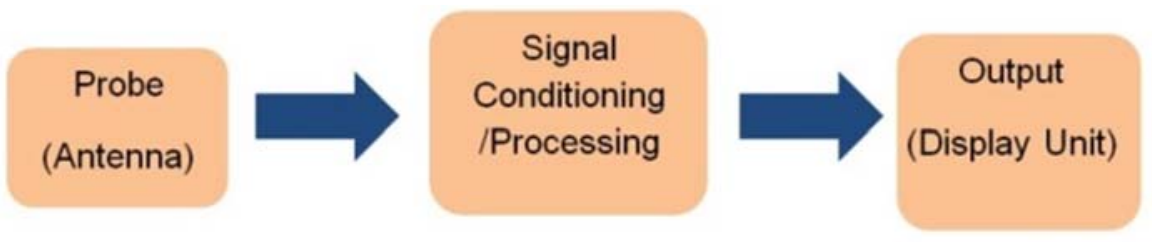

Figure 1. Block diagram showing the stages of the EM field detector circuit.

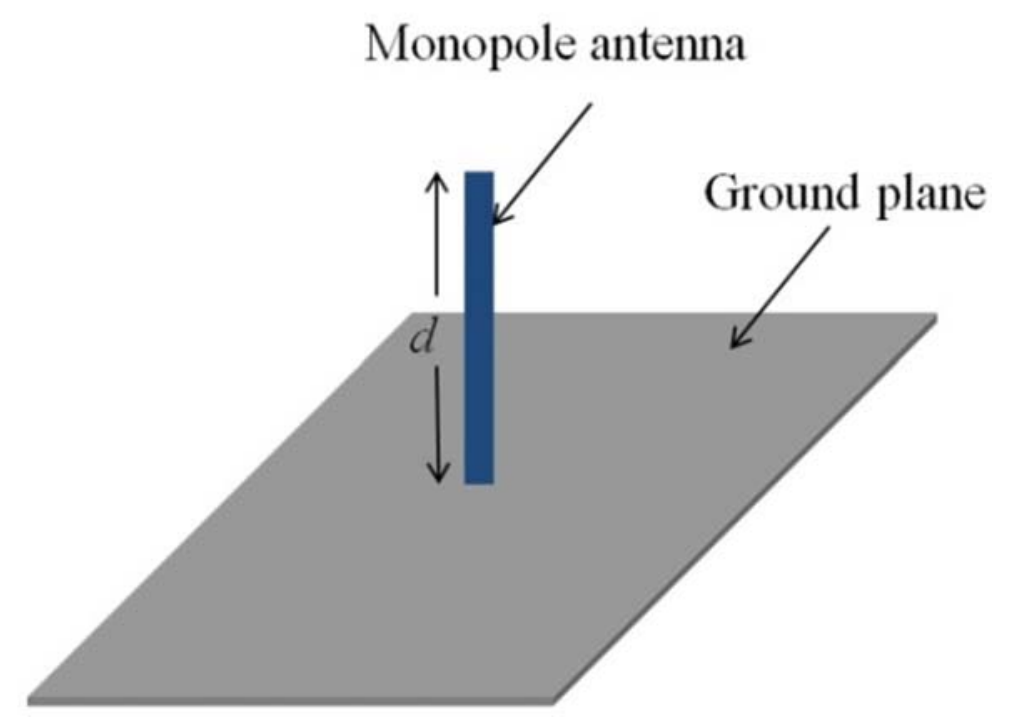

Figure 2. A quarter-wavelength monopole antenna mounted on top of a metallic chassis.

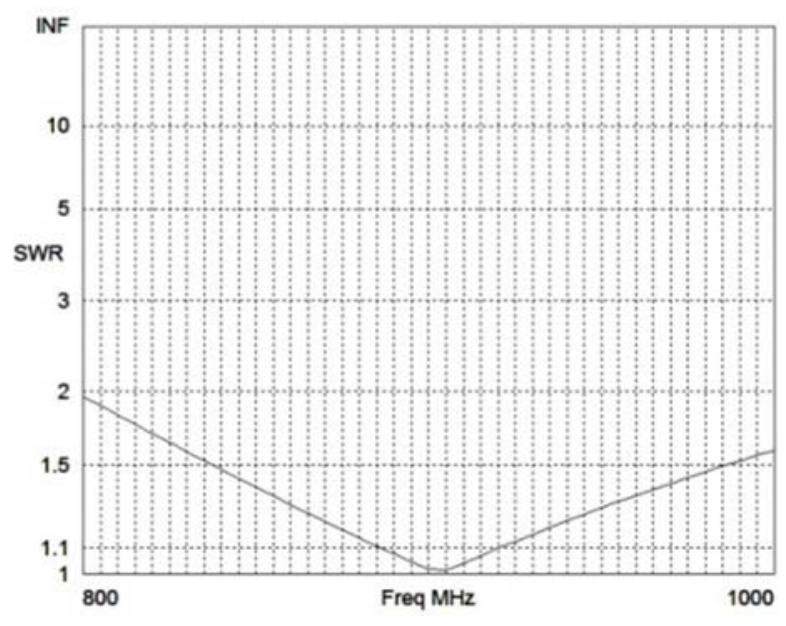

Figure 3. Voltage standing wave ratio for the monopole antenna resonating at the $900 \mathrm{MHz}$ band. 


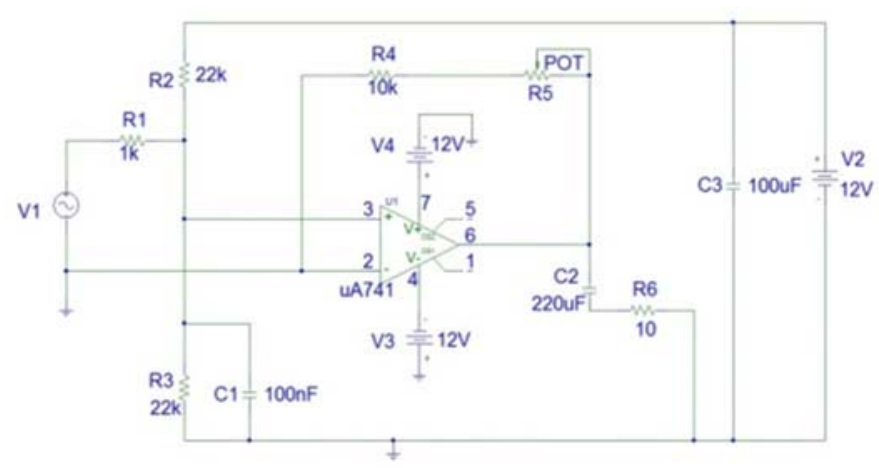

Figure 4. The detector circuit as modeled in Pspice to sense any possible EM field radiated emissions from $60-\mathrm{Hz}$ power meters.

The circuit was modeled and simulated using Pspice circuit simulator. An operational Amplifier (UA741) was used and found suitable for such extremely low-frequency applications. The detecting circuit needs only a DC supply of 12 volts. The output is then captured and recorded. Both the input and output signals out from the simulation model are shown in Fig. 5, while experimental results are displayed in Fig. 6.

The output signal captured by the detector circuit has a frequency of $60 \mathrm{~Hz}$, which was the original input signal to the circuit. Note that the output waveform is captured at the output waveform is captured at the output terminals of the general-purpose operational amplifier. In fact, some ripples were noticed and appeared at the output terminal of the detector circuit as shown in Fig. 6. Such ripples are attributed to the power line signal that was picked up by the detector circuit. Nonetheless, the $60-\mathrm{Hz}$ input signal was detected at the output of the detector circuit, despite the small coupling that was observed due to the EM wave radiated emissions from the power line cords in laboratory. This observation of electromagnetic interference issues has been clarified in section 1 . such coupling effect is attributed mainly to the magnetic field of radiated emissions from the power line cords which was inherently induced on the detector circuit.

\subsection{Detectors for Extremely Low-Frequency Electronic Circuits}

In this part, we examine the ability of the detector circuit to capture electromagnetic waves at the extremely low-frequency range which is of interest in electronic circuits troubleshooting. Figure 7 depicts another detecting circuit for low- frequency applications, targeting high electromagnetic exposure from extremely lowfrequency electronic devices and systems. In this circuit, a 9-V DC supply was found sufficient. The experimental setup along is illustrated in Fig. 8. For demonstration purposes, the circuit was implemented in general-purpose breadboard.

The captured output signal is shown in Fig. 9, when an input waveform at a frequency of 1.0 $\mathrm{MHz}$ was considered. It is worth noting here that other signals of higher frequency up to $3.5 \mathrm{MHz}$ were easily captured by the detecting circuit, beyond which noise started to appear at output waveform. This is attributed to the limited capabilities of the general-purpose transistor and operational amplifier.

\subsection{Detectors for Mobile Phones and WiFi Access Points}

In this part, the detector circuitry, as shown in Fig. 10, is developed to predict potential electromagnetic radiation from mobile devices and WiFi access points simultaneously. In order to accomplish this, a dual-band monopole antenna system is designed to capture the energy of such incoming waves of the dual-resonance system.

Figure 11 shows the simulation results when considering an AC signal with a frequency of 900 $\mathrm{MHz}$. Both input and output signals are shown. It can be seen that the circuit was able to identify the $900 \mathrm{MHz}$ signal, as evident from the period of the output waveform.

Figure 12 shows the implemented circuit (see Fig. 10) in breadboard. In the implemented detector circuit, a single high-profile monopole antenna was used, where it was designed to resonate and capture the energy of $900 \mathrm{MHz} \mathrm{EM}$ 

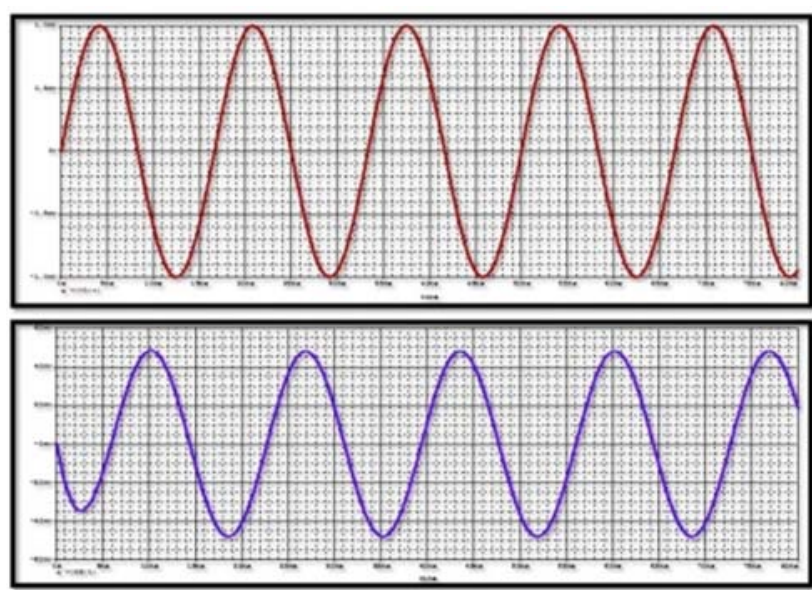

Figure 5. The detector circuit as modeled in SPICE to sense any possible EM field radiated emissions from $60-\mathrm{Hz}$ power meters.

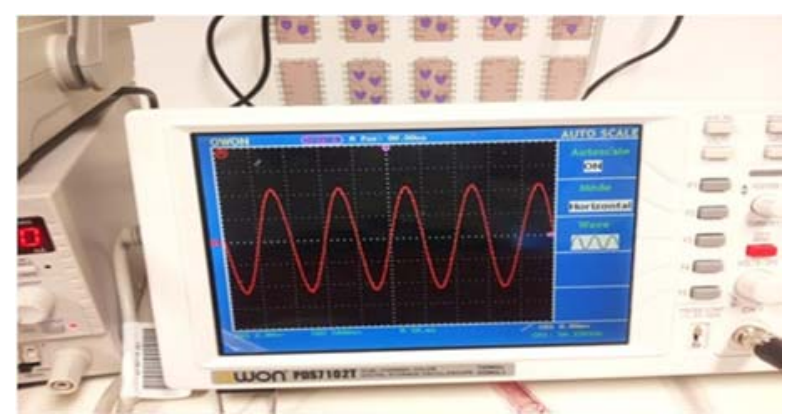

(a)

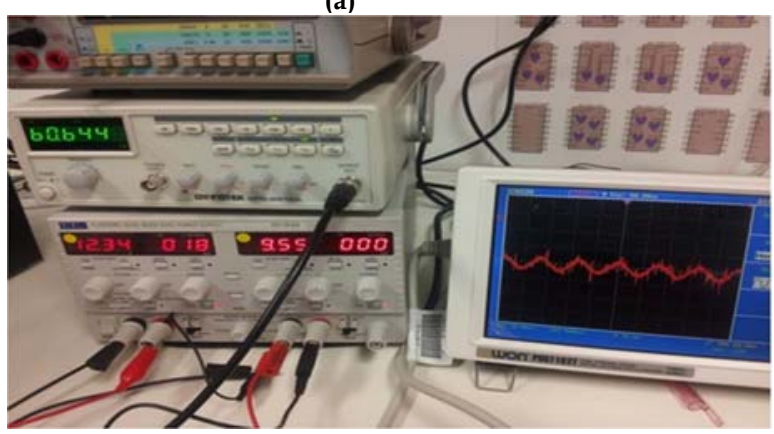

(b)

Figure 6. Experimental setup for the $60-\mathrm{Hz}$ detector circuit (a) input waveform; and (b) captured signal at output terminals of the detector circuit.

wave radiated out of mobile phones. Both LED as well as a qualitative EM field strength display unit will turn on once a call/text message is either transmitted or received.

Interestingly, the EM field strength display unit was highly sensitive to incoming signals from either mobile phone or WiFi router, which is indicated by the levels in the display unit (see Fig. 12). In order to avoid any sources of external electromagnetic interference, the same circuitry has been built in the lab using printed circuit board (PCB) technology. 


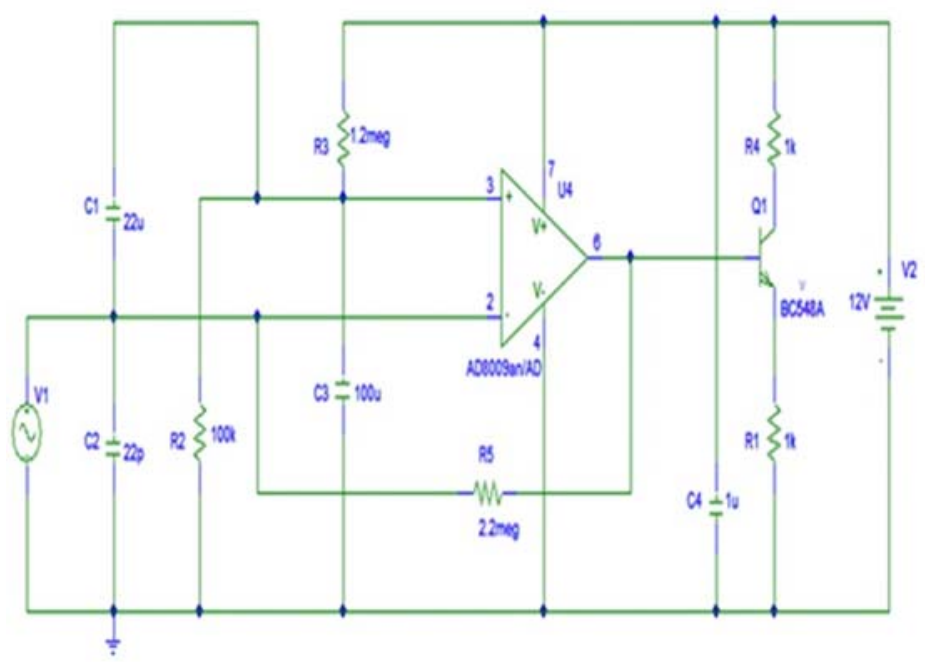

Figure 7. Detecting circuit that detects electromagnetic exposure from low-frequency electronic devices.

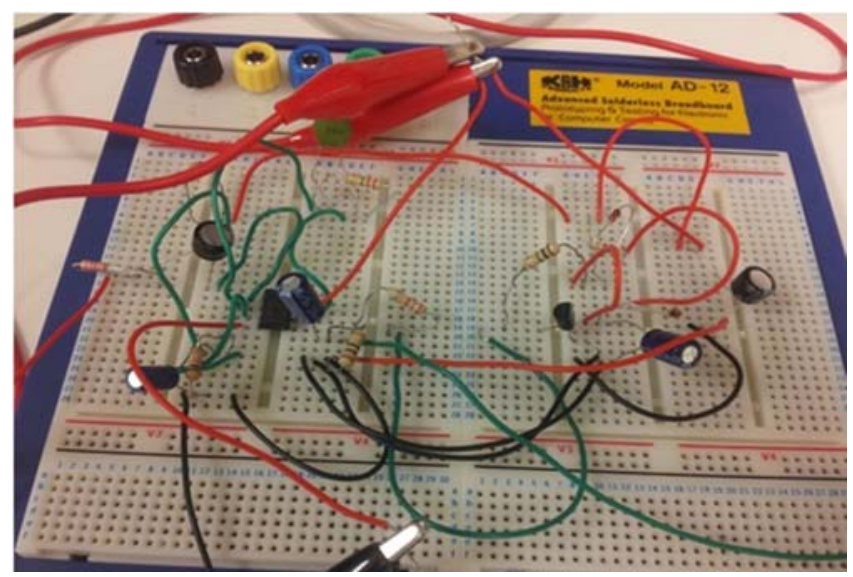

Figure 8. Experimental setup of detecting circuit for low-frequency applications.

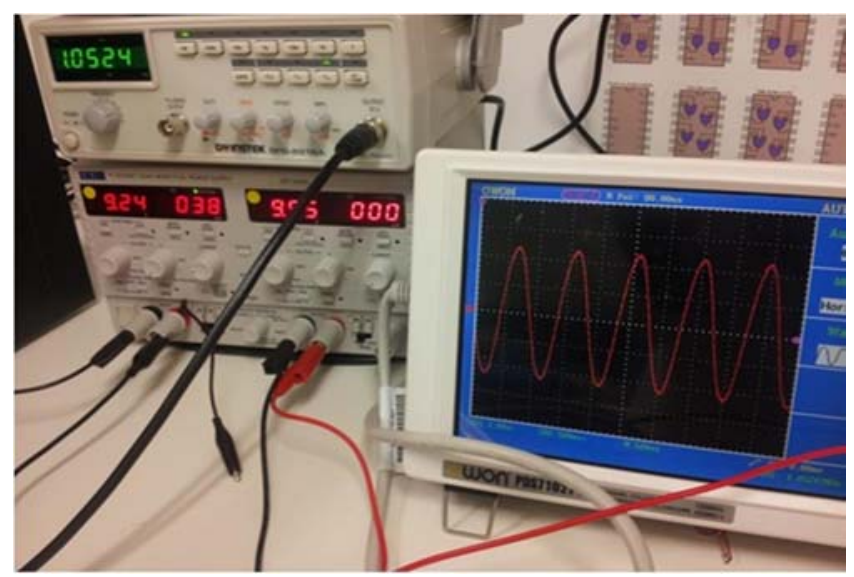

Figure 9. Measured output waveform of the low-frequency detecting circuit at $1.0 \mathrm{MHz}$, visualized by an oscilloscope. 


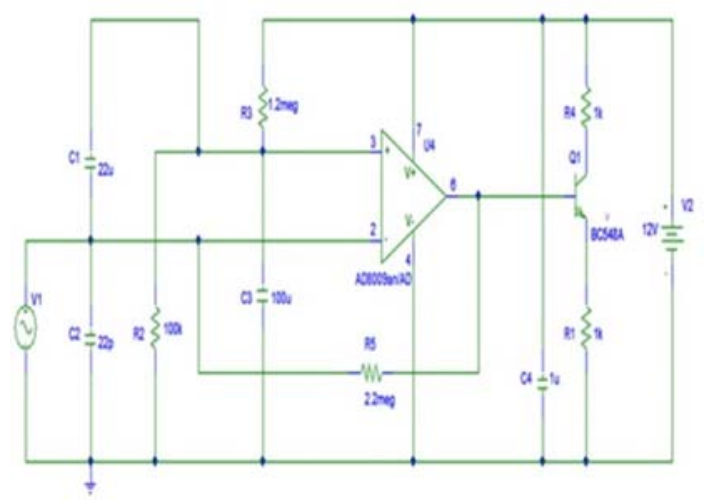

Figure 10. Detecting circuit that detects EM radiation from mobile phones and WiFi access points, simultaneously through the use of a dual-band monopole antenna.
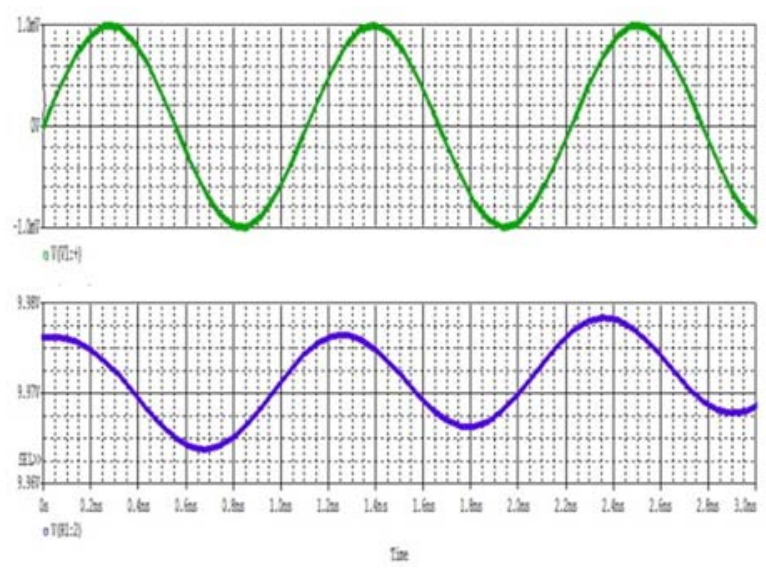

Figure 11. The simulation results for the detecting circuit modeled in Pspice. Note that input waveform is shown on top, whereas the output is on bottom.

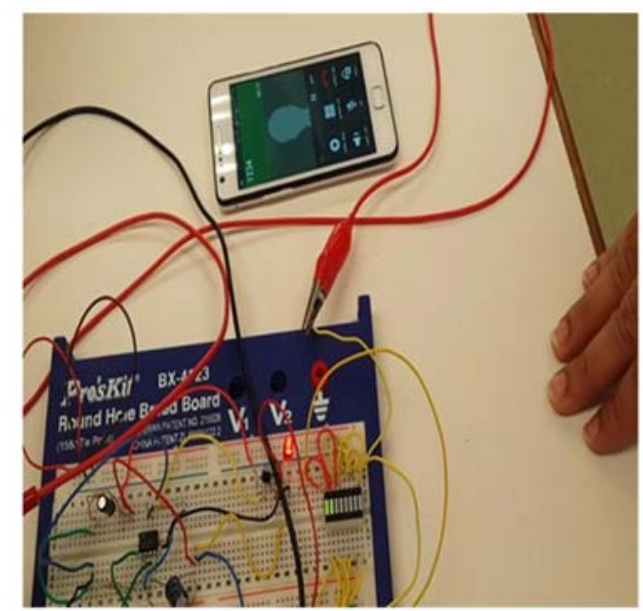

Figure 12. The built-in detecting circuit in breadboard with a single monopole antenna. Notice the blinking of the LED as well as strength display unit being on when a call was either placed or received. 
Figure 13 shows details of the detector circuit implemented on $\mathrm{PCB}$, where two dual-antenna elements were mounted at front end of the detecting circuit in order to simultaneously detect EM radiation coming from either the $900 \mathrm{MHz}$ GSM band or wireless devices at $2.45 \mathrm{GHz}$.

It is instructive to note here that the selection of the lumped elements for the detector circuit in part (c), for instance, was not straight forward. In fact, the selection was done carefully through series of a trained "trial-and-error" procedure, as some of the components were found to be quite sensitive to the operating frequency of the incoming EM wave, including the use of specialized transistors and operational amplifiers to fit the operating frequency regime. For instance, the general purpose operational amplifier was found not suitable for this kind of applications as frequency is relatively high. Special amplifiers, LM358 and AD-845, were employed in the detectors circuits of parts (b) and (c) (LM358 Datasheet), although other alternative wideband amplifiers could be used. Furthermore, a generic 2N2222 NPN transistor was used for the circuits in parts (a) and (b), while a special transistor, BC548 (BC548 Datasheet), that fits highfrequency applications was adopted in the detector circuit of part (c).

Several sensitivity analyses were also conducted in laboratory in order to quantify the sensitivity of the antenna elements to EM radiation. It was found out that the detector targeting mobile phone devices can detect EM radiation up to almost 1.0 meters, which is quite useful as it reasonably detects within both nearand far-field regime from the EM source of radiation. However, it was observed that the detector system is highly sensitive to EM radiation within near-field proximity to the source of radiation as compared to the far-field region from the same source of radiation. The results of this study are summarized and tabulated as presented in Table. 1. For this study, the energy of EM waves radiated out from mobile devices were captured and detected when either transmitting or receiving calls. As expected, EM field strength was decreased as we get away from the source of radiation.
Figure 14 shows the detected mobile phone signal by the developed dual-band probe. The detected signal strength was around $915 \mathrm{MHz}$. In this particular case, only mobile phone was in vicinity of the detecting probe where no WiFi signals were in close proximity. Hence, a peak that falls around the $900 \mathrm{MHz}$ band is visible in the Spectrum Analyzer.

Based on the accomplished simulated and measured results, there are issues that need to be addressed here. Due to the sensitivity of the detector circuits to operating frequency of the incoming electromagnetic waves, several prototypes were developed to fit three frequency energy, covering real-world applications, ranging from as low as power line meters, to mobile phone devices and several bands of telecommunication services. In future, it will be essential to come up with a wideband detecting circuitry that can fit a wide spectrum of energy applications. In addition to that, incorporating an EM field strength meter is essential in order to provide a quantitative measure of EM radiated emissions strength from potential sources of interference. Furthermore, the use of high-profile antennas may not fit other applications, especially for biomedical applications, eg. implanted devices. Therefore, the use of low-profile miniaturized antennas yet being wideband are highly desirable. The aforementioned issues will be the subject of future work.

Table 1. Measured alternating current voltage and current at output stage of the detecting system at various distances from mobile phone unit.

\begin{tabular}{ccc}
\hline $\begin{array}{c}\text { Distance } \\
(\mathrm{cm})\end{array}$ & $\begin{array}{c}\text { Voltage } \\
(\mathrm{V})\end{array}$ & $\begin{array}{c}\text { Current } \\
(\mathrm{m} \boldsymbol{A})\end{array}$ \\
\hline 0 & 2.3 & 5 \\
14 & 1.5 & 5 \\
30 & 1.2 & 2 \\
40 & 1.1 & 2 \\
50 & 0.8 & 2 \\
60 & 0.6 & 1 \\
80 & 0.5 & 1 \\
100 & 0.3 & 1 \\
$>100$ & 0 & 0 \\
\hline
\end{tabular}



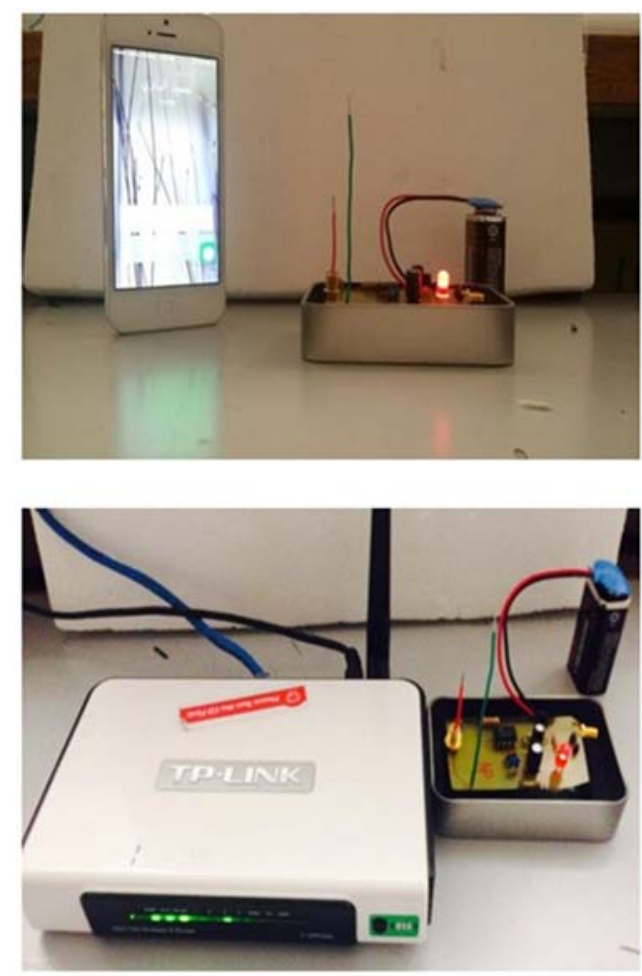

Figure 13. The detector system with an integrated dual-band monopole antenna, as shown in both images. Details of two applications of detecting EM waves generated from both mobile phone (top image) and WiFi access points (bottom snapshot) are shown.

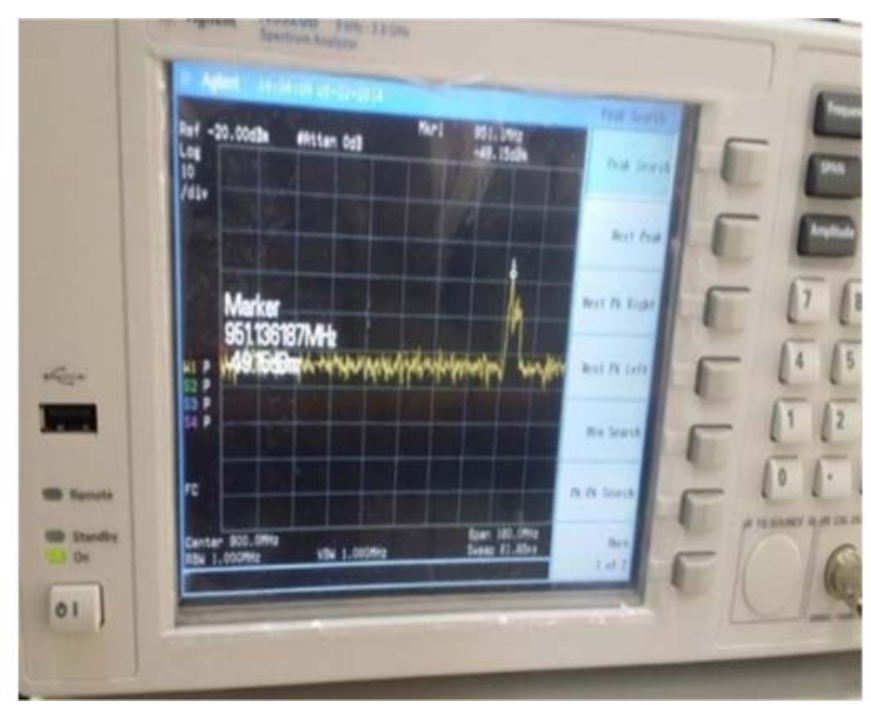

Figure 14. The detected EM radiation out from a mobile phone using the spectrum analyzer. 


\section{Conclusions}

In this research work, low-frequency electromagnetic field detector system was developed, numerically simulated and experimentally validated. The proposed detector was integrated with a dual-band monopole antenna system that targets detection of electromagnetic radiated emissions from both mobile phone units (GSM-900 MHz band) and wireless devices (WiFi access points) operating at the $2.45 \mathrm{GHz}$ band. The prototype was simulated, and validated against experimental data for the aforementioned applications. The measured results agree with expected numerical results. Other prototypes were presented that can easily predict and sense possible malfunctions in power line meters as well as EM interference threats in highspeed printed circuit boards.

Although the incorporated antenna system is narrowband, the dual-band antenna elements were quite sensitive to capture the electromagnetic radiated emissions from low-energy devices as evident from the experimental prototype (see Fig. 12). The use of wideband antenna system is useful for a standalone monitoring probe system that will find useful applications, for instance for mobile communication service provides as a test drive scanning tool, or as an alarming system for diagnosis and industrial troubleshooting for potential EMI threats.

\section{Acknowledgments}

The author would like to thank Sultan Qaboos University for providing the financial resources needed for the project. The author would also like to thank Issa Al-Balushi, Mohammed Al-Alawi, Mohammed Al Sadairi, and Salim Al-Risi for carrying out couple of measurements for this work.

\section{References}

Advisory Group on Non-ionizing Radiation (1992), Electromagnetic fields and the risk of cancer report. National Radiological Protection Board 3(1):1-138.

Advisory Group on Non-ionizing Radiation (1994), Health effects related to the use of visual display units report. National Radiological Protection Board 5(2):1-75.
Archambeault B, Ramahi O, Brench C (2001), EMI/EMC computational modeling handbook, $2^{\text {nd }}$ ed, Boston: Kluwer.

Bait-Suwailam M, Alavikia B, Ramahi O (2014), Reduction of electromagnetic radiation from apertures and enclosures using electromagnetic bandgap structures. IEEE Transactions on Components, Packaging, and Manufacturing Technology 4(5):929-937.

Bait-Suwailam M, Al-Balushi I, Al-Sadairi M, AlAlawi M, Al-Risi S (2014), Electromagnetic radiation probe using a detector circuit with a dual-band antenna system. Proceedings of the $2^{\text {nd }}$ International Conference on Electronics Design (ICED), Penang, Malaysia.

Balanis C (2005), Antenna theory: Analysis and design $3^{\text {rd }}$ ed. Hoboken, NJ: John Wiley, Inc.

Bernardi P, Cavagnaro M, Pisa S, Piuzzi E (1998), SAR distribution and temperature increase in an anatomical model of the human eye exposed to the field radiated by the user antenna in a wireless LAN. IEEE Transmission on Microwave Theory and Techniques 46(12):2074-2082.

Brench C, Brench B (1989), Effects of cable and peripheral placement on radiated emissions. Proceedings of the IEEE National Symposium on Electromagnetic Compatibility May 23-25, 351-356.

Buchanan WJ, Gupta NK, Arnold JM (1994), Application of 3D finite-difference time-domain (FDTD) method to predict radiation from a PCB with high speed pulse propagation. Proceedings of the Ninth International Conference on Electromagnetic Compatibility, University of Manchester 287-291.

Datang Telecom Technology \& Industry Group (2013), Evolution, convergence, and innovation-White paper on 5G technology. Retrieved from: www.datanggroup.cn/ upload/accessory/201312/201312919445526537 2.pdf.

Fairchild (2014), BC546/BC547/BC548/ BC549/ BC550 NPN epitaxial silicon transistor. Retrieved from: hwww.fairchildsemi.com/ ds/ BC/BC547.pdf.

Hanna S, Motai Y, Varhue W, Titcomb S (2009), Very low-frequency electromagnetic field detector with data acquisition. IEEE Transmission on Instrumentation and Measurement 58(1):129-140.

International Commission on Non-Ionizing Radiation Protection (1998), Guidelines for 
limiting exposure to time-varying electric, magnetic and electromagnetic fields (up to 300 GHz). Health Physics Journal 74(4):494-522.

IEEE Standard Std. (1994), Procedures for measurement of power frequency electric and magnetic fields from AC power lines. IEEE Std 644. DOI: 10.1109/IEEESTD. 1995. 122621.

Lin J (2009), Specific absorption rates (SAR) induced in head tissues by microwave radiation from cell phones. IEEE Antennas and Propagation Magazine 42(5):138-140.

Mann J, Lee R, Aragon T, Bhatia R (2001), Radio frequency radiation from broadcast transmission towers and cancer: A review of epidemiology studies. Retrieved from: www.sfdph.org/dph/ files/reports/ Studies Data/ RadioFreqRadRpt032001.pdf.

Park H, Park H, Lee H (2013), A simple method of estimating the radiated emission from a cable attached to a mobile device. IEEE Transmission on Electromagnetic Compatibility 55(2):257-264.
Rowley J, Waterhouse R (1999), Performance of shorted microstrip patch antennas for mobile communications handsets at $1800 \mathrm{MHz}$. IEEE Transmission on Antennas and Propagation 47(5):815-822.

Seabury D (2005), An update on SAR standards and the basic requirements for SAR assessment. Retrieved from: www.ets-lindgren.com/pdf/ sar_lo.pdf.

Texas Instruments (2014), LMx58-N Low-Power, Dual-Operational Amplifiers. Retrieved from: http://www.ti.com/lit/ds/symlink/lm158n.pdf.

Ulaby FT, Michielssen E, Ravaioli U (2014), Fundamentals of Applied Electromagnetics $6^{\text {th }}$ ed. London: Pearson Education Limited 30-31.

World Health Organization (2011), Electromagnetic fields and public health: Mobile phones. Fact sheet No. 193. Retrieved from: http://www.who.int/mediacentre/factsheets/f s193/en/. 